\title{
Cuidados de enfermagem no puerpério
}

\section{Cuidados de enfermería en el puerpério}

\section{Nursing care in the puerperium}

Ana de Jesus Gomes de Oliveira ORCID: https://orcid.org/0000-0002-2434-5914 Universidade de Palmas, Brasil E-mail: anajesusg@gmail.com

Patrícia Silva Barros

ORCID: https://orcid.org/0000-0001-5242-3951 Universidade Federal do Maranhão, Brasil

E-mail: patty.barross@hotmail.com

Raquel Pereira Souto Matos

ORCID: https://orcid.org/0000-0002-9406-8879 Unidade de Ensino Superior Sul do Maranhão, Brasil

E-mail: soutonsp@gmail.com

Nilcelete dos Santos Vieira

ORCID: https://orcid.org/0000-0002-5190-3758 Universidade Salgado de Oliveira, Brasil E-mail: nilcelete@msn.com

Rodrigo Teles de Medeiros Melo

ORCID: https://orcid.org/0000-0001-6683-0590 Faculdade de Medicina de Juazeiro, Brasil

E-mail: rodrigotelesmm@ outlook.com

Thiago Teles de Medeiros Melo

ORCID: https://orcid.org/0000-0001-6310-7057 Instituto Tocantinense Presidente Antônio Carlos, Brasil

E-mail: telesmm@hotmail.com

Raylton Aparecido Nascimento Silva

ORCID: https://orcid.org/0000-0003-3832-7685

Universidade Federal de Santa Maria, Brasil E-mail: rayltonaparecido@gmail.com

Tamyze Bezerra Gomes

ORCID: https://orcid.org/0000-0002-3698-4158

Universidade de Palmas, Brasil

E-mail: tamyzegomes@gmail.com

Vitor Pachelle Lima Abreu

ORCID: https://orcid.org/0000-0001-9065-3272

Universidade Federal do Tocantins, Brasil E-mail: vpachelle@gmail.com

Thiago Oliveira Sabino Lima

ORCID: https://orcid.org/0000-0003-2677-9481 Universidade de Palmas, Brasil

E-mail: thiagosabino@uft.edu.br

Ruhena Kelber Abrão

ORCID: https://orcid.org/0000-0002-5280-6263

Universidade Federal do Tocantins, Brasil

E-mail: kelberabrao@gmail.com

\section{Resumo}

Abordar sobre a assistência por parte da equipe de Enfermagem durante o ciclo reprodutivo feminino, aspectos da saúde da mulher durante a fase puerperal é o objetivo deste trabalho. Diante disso foi realizada uma revisão de literatura narrativa, qualitativa com busca por artigos científicos nas bases de dados Bireme, SCielo e PubMed. Como resultados, identificamos que a assistência de enfermagem a puérperas reflete em satisfação às pacientes, que se sentem confortáveis e confiantes sob os cuidados da enfermagem No entanto ainda há uma inclinação ao abandono dos cuidados, pelas puérperas, após o parto, visto que a recusa da revisão pós-parto ainda é uma realidade comum. Cabe ao estudo novas pesquisas, principalmente na lacuna de educação em saúde as gestantes sobre os seus direitos e que estes não acabam no momento do parto, e quanto aos profissionais é importante que haja uma capacitação continuada sobre a assistência no puerpério, visando melhorar cada dia mais os cuidados ofertados a esse público.

Palavras-chave: Cuidados de enfermagem; Puerpério; Saúde da mulher. 


\begin{abstract}
This study aims at a compilation of the assistance provided by the nursing team during the period of the female reproductive cycle, aspects of women's health during the puerperal phase. From this, a narrative literature review was carried out, in view of the search for scientific articles in the Bireme, SCielo and PubMed databases. How to identify nursing care for puerperal women, still reflecting compliance with patients, who feel comfortable and confident under nursing care after childbirth rejection There is no inclination to abandon patients given that the results of the review postpartum is still a common reality. It is up to the study to further research, especially in the education gap in pregnant women about their children and who do not end at the time of delivery, and as for those, it is important that there is continued health on the rights of the puerperium, to improve more and more the care offered to women. this audience.
\end{abstract}

Keywords: Nursing care; Puerperium; Women's Health.

\title{
Resumen
}

Este estudio tiene como objetivo recopilar la asistencia prestada por el equipo de enfermería durante el período del ciclo reproductivo femenino, aspectos de la salud de la mujer durante la fase puerperal. A partir de ello, se realizó una revisión bibliográfica narrativa, de cara a la búsqueda de artículos científicos en las bases de datos Bireme, SCielo y PubMed. Cómo identificar el cuidado de enfermería a la mujer puérpera, aún reflejando el cumplimiento de las pacientes, que se sienten cómodas y confiadas bajo el cuidado de enfermería después del rechazo del parto. No hay inclinación a abandonar a las pacientes dado que los resultados de la revisión posparto aún es una realidad común. Corresponde al estudio seguir investigando, sobre todo en el desfase educativo de las mujeres embarazadas sobre sus hijos y que no terminan en el momento del parto, y en cuanto a esas, es importante que se mantenga la salud sobre los derechos de la puerperio, para mejorar cada vez más la atención que se ofrece a las mujeres de este público.

Palabras clave: Atención de enfermería; Puerperio; Salud de la mujer.

\section{Introdução}

No tocante à sexualidade e contracepção feminina, as mesmas são vistas como a maior responsabilidade da mulher, sendo socialmente responsabilizada pelos cuidados contraceptivos. Uma vez que estas não atendem a esses “deveres”, são destinados a gestações indesejáveis, abortos clandestinos e ao discurso que as responsabilizam e as culpabilizam. A mulher sofre também com a responsabilidade que quase sempre é unilateral de cuidar dos filhos e do lar (Rodrigues et al, 2021).

A mulher inicia seu período fértil após a menarca e finaliza com a menopausa, e ao longo desse ciclo reprodutivo, ocorrem vários fenômenos como ciclo ovárico e ciclo uterino que sofrem as ações hormonais do folículo estimulante (FSH), luteinizante (LH) que são sintetizados no cérebro e os hormônios estrogênio e progesterona sintetizados nos ovários (Durval et al, 2016).

Logo após o parto, com a saída da criança e da placenta, a mulher inicia o seu período puerperal. Que pode ser classificado de imediato - do $1^{\circ}$ ao $10^{\circ}$ dia, tardio - do $10^{\circ}$ ao $45^{\circ}$ dia e remoto - após o $45^{\circ}$ dia. Há definições a serem compreendidas quanto ao fim do puerpério, sendo que descritas com seis a oito semanas após o parto, período em que as modificações anatômicas e fisiológicas do organismo materno, em especial do seu aparelho reprodutor, são marcadamente notadas (Santos et al, 2018, Santos et al, 2021)

Durante o puerpério, a mulher está exposta a diversas transformações em seu corpo, com risco de exposição à maior frequência de agravos que são causas específicas de morbimortalidade materna. O Programa de Humanização do Pré - Natal e Nascimento (PHPN) instituído pelo Ministério da Saúde recolocam em pauta a garantia do acesso e a qualidade do cuidado no ciclo gravídico-puerperal corroborando com um dos princípios do SUS, que é a Integralidade (Saúde, 2012).

Hoje, muito já se conquistou acerca dos direitos da puérpera. O seu parceiro tem direito à licença paternidade de 5 dias a contar da data do parto; a gestante tem direito à licença maternidade de 120 dias e o salário integral a partir do $8^{\circ}$ mês de gestação e a mulher tem o direito de se ausentar do local de trabalho pelo menos duas vezes ao dia, por pelo menos 30 minutos, para a amamentação até que o bebê complete 6 meses de vida (Santiago et al, 2012).

A saúde da mulher durante o Puerpério é essencial, sendo uma atribuição dos profissionais de saúde a partir da Estratégia Saúde da Família (ESF) e podem-se destacar grupos que são prioritários para atendimento domiciliar, no qual se 
enquadra a atenção domiciliar, haja vista que, o cuidado durante o puerpério deve ser imediato, pois visa a uma assistência individual e holística, estabelecendo vínculo de confiança com a puérpera e toda sua família (Medeiros, 2016).

A puérpera também tem direito à consulta médica e de enfermagem dentro do período puerperal para que seja avaliada sua saúde pós-parto e reforçada as orientações durante essa fase. Além disso, com as altas incidências da depressão pós-parto, o SUS oferta serviço de apoio psicológico para as gestantes que não estejam adaptadas à realidade da maternidade (Côrrea; et.al., 2017).

A enfermagem destaca-se pelas suas ações em cuidado, devendo assegurar que os pacientes possam gerir sua própria saúde e capacitá-los para o autocuidado. Quanto a saúde da mulher é importante que esta seja capacitada em seu autocuidado, em práticas corretas frente à gestação, o puerpério, o aleitamento materno, o planejamento reprodutivo, a prevenção de IST, controle do câncer ginecológico e cuidados quanto ao climatério (Riul; et.al, 2018, Rocha et al, 2020).

Nesse sentido, o presente estudo tem como objetivo realizar uma análise de conhecimentos acerca da fase puerperal e a assistência por parte da equipe de Enfermagem. Portanto, esse estudo demonstra a importância da análise da literatura para tal temática, bem como elucidar questões que permitirão entender melhor seus benefícios para produção do saber no campo pessoal, profissional e social, a fim de desenvolver ações que viabilizem a melhoria do processo de trabalho.

\section{Revisão bibliográfica}

Historicamente no Brasil no século XIX, os partos e seus cuidados eram realizados por mulheres conhecidas popularmente como aparadeiras, comadres ou mesmo de parteiras-leigas. Desconheciam do saber empírico e assistiam domiciliam entre às mulheres durante a gestação, parto e puerpério como também nos cuidados com o recém-nascido (Brenes, 1991).

A Organização Mundial de Saúde (OMS) define que para uma condição de saúde, o ciclo gravídico puerperal é uma circunstância na vida da mulher, que se segue a um período de fertilidade, podendo ser desejada e planejada, ou surpreendendo de maneira não prevista ou planejada, com sentimentos de ambivalência e de aceitação, ou não (Saúde, 2020).

A primeira definição clara sobre os direitos sexuais e reprodutivos foi instituída na Conferência Internacional de População e Desenvolvimento, onde foram abordados e evidenciados os direitos da mulher quanto ao planejamento familiar, podendo decidir sobre a maternidade, quantos filhos desejam ter e o momento certo para a concepção, devendo esta ser livre de qualquer tipo de descriminação ou violência e ainda quanto o direito a pratica de relações sexuais de forma segura, com prevenção a gravidez indesejada, infecções sexualmente transmissíveis, HIV/AIDS, e ao direito a educação sexual e reprodutiva (Justino; et. al., 2019; Rodrigues et al, 2020).

Na literatura é possível verificar variados conceitos acerca da saúde da mulher com relevância a biologia e anatomia corporal feminina e outros afirmam seus direitos e questões relacionadas à cidadania. Ao avaliar aspectos mais restritos, o corpo feminino é visto apenas em relação à reprodução e para a mulher atribui-se apenas o seu dever com a maternidade. A saúde feminina é limitada à saúde materna ou à ausência de enfermidade que esteja associada ao processo da reprodução humana (Coelho, 2003).

Os direitos sexuais e reprodutivos das mulheres são prioridades quanto o planejamento de políticas públicas, sendo dever do Ministério da Saúde planejar e programar propostas que abranja a educação sexual, os cuidados com o corpo, os direitos reservados a mulher quanto a sexualidade e reprodução e ainda capacitação dos profissionais de saúde para que a assistência seja efetiva e integral, na promoção, prevenção e tratamento da saúde (Saúde, 2004).

As mulheres não sabem sobre seus direitos quanto à escolha da quantidade de filhos que deseja ter e quando tê-los, não sabem sobre a disponibilização gratuita de preservativos e contraceptivos pela rede pública de saúde e também ao direito à 
educação sobre infecções transmissíveis e gravidez, e principalmente que estes direitos não devem ser negados e nem forçados, livres de violência e imposições (Marques, 2011).

Também é reservado à mulher o direito ao acompanhamento da gestação, devendo este ser oferecido de forma gratuita pelo Sistema Único de Saúde (SUS), especificamente na Atenção Primária, com a realização de consultas mensais, ou de acordo com a necessidade, realização de exames para avaliar a saúde da mãe e do feto, como laboratoriais e de ultrassom, atendimento humanizado no momento do parto, com direito a acompanhante no parto e no alojamento conjunto, direito a contato imediato com o filho após o nascimento e amamentação, direitos esses enfatizados pela Rede Cegonha, e ainda o direito ao acompanhamento após o parto, tanto da mulher quanto do bebê (Jorge et al., 2015).

\subsection{Ciclo Reprodutivo Feminino}

O ciclo reprodutivo feminino compreende o período de tempo entre a menarca, que é a primeira menstruação da mulher, até a menopausa, que é a cessação da menstruação. A puberdade, ocorrente na adolescência, marca o início de alterações hormonais que são responsáveis por mudanças físicas, psicológicas e emocionais, nas meninas essas mudanças repercutem no aumento das mamas, aumento de pelos pelo corpo, em especial os pubianos e das axilas, alterações na voz, dentre outras alterações, a nível fisiológico, nessa fase a menina passa por uma reativação na interação - entre hipotálamohipófise-ovários - que propicia a menarca e o início do ciclo reprodutivo, sendo o ciclo menstrual como protagonista dessa (Bouzas et al, 2010).

O ciclo menstrual tem em média 28 dias, e é dividido em fases, que são determinadas pela liberação de hormônios como estrogênio e progesterona. A primeira é a fase folicular, onde ocorre a produção de hormônio folículo estimulante propiciando a maturação do folículo, esse mesmo folículo é responsável pela produção de estrogênio que provoca o espessamento do endométrio. Quando chega a sua fase madura, cerca de 14 dias após o primeiro dia da menstruação, ocorre a ruptura do óvulo maduro, a segunda fase conhecida por ovulação, é nesse momento em que ocorre o período fértil, e a mulher está receptiva a fecundação, caso isso ocorra o zigoto se fixará ao útero, ocorrendo a produção de uma glicoproteína que mantém o corpo lúteo, evitando a menstruação. Caso não ocorra a fecundação o corpo sexual lúteo para de secretar progesterona e estrogênio o que ocasiona a descamação do endométrio que é caracterizada pela menstruação (Teixeira et al, 2012).

Após a menarca, que ocorre entre os 15 , podendo ser precoce ou tardia a essas idades, a mulher pode ter um ciclo menstrual que dura por 35 a 40 anos, e são acompanhados de sintomas pertinentes a cada fase do ciclo, como alterações de humor, dores localizadas, nas mamas e pernas, cólicas, especialmente no período menstrual, dores de cabeça, calor, aumento da libido, fadiga, dentre outros (Saúde,2008). É possível que, por diversos fatores a menarca seja um acontecimento cada vez mais precoce, onde meninas ainda na infância podem vivenciá-la, e os fatores relacionados a isso podem ser tanto genéticos, sociais, ambientais, climáticos, nutricionais e alguns outros (Carvalho et al., 2007).

Outro marco importante no ciclo reprodutivo feminino é a sexarca, ou primeira relação sexual, caracterizada pela ruptura do hímen, uma membrana fina localizada no canal vaginal, e marca o início da vida sexual ativa de uma mulher. Contudo, muitas vezes esse primeiro contato sexual não está relacionado à informação e segurança, especialmente quando ocorre na adolescência, o que pode significar uma maior vulnerabilidade para infecções sexualmente transmissíveis (IST) ou gravidez indesejada (Santos et al., 2014)

O ciclo reprodutivo feminino se finaliza com a chegada do climatério, conhecido popularmente como menopausa, esta fase é ocorrente nas mulheres de meia idade, entre 55 a 65 anos, podendo ocorrer também de forma precoce. Por ser caracterizado como a ausência de liberação de óvulos para fertilização, o climatério pode ocorrer também por meio de intervenções cirúrgicas, como o ooforectomia bilateral, associada, ou não, a histerectomia (Valença et al, 2010). 
Contudo, é importante evidenciar que essa fase, climatério, não corresponde somente a finalização do ciclo reprodutivo, e da menstruação, mas agrega também mudanças emotivas, físico e psicossociais, variando de mulher para mulher. Entende-se assim, que há vários fatores que podem influenciar nas mudanças sofridas pelas mulheres na fase do climatério, como: meio de vida ao qual está inserida, vida sexual, histórico de paridade, hábitos alimentares, acesso à saúde e informações, conflitos em vários âmbitos da vida e ainda conhecimento sobre sua sexualidade (Saúde, 2008).

Devido à ausência do ciclo menstrual, característico da menopausa, a mulher passa a produzir pouco ou nenhum dos hormônios envolvidos nesse ciclo, progesterona e estrogênio, portanto é recomendável a reposição hormonal, evitando que ela passe por todas as inconveniências relacionadas a pele, libido, funcionalidade física, dentre outros (Oliveira et al., 2016).

\subsection{Gestação}

A gravidez está caracterizada como condição para a sobrevivência da vida humana, tornando indispensável à renovação geracional, e representa o período de formação de um novo ser. A fase nova que pode perdurar um período que dura cerca de 40 semanas que começa pela concepção, e termina com o parto, é um período em que ocorrem alterações profundas no que respeita ao estilo de vida, favorecendo transformações não apenas na vida pessoal, mas também na vida do casal e de toda a família. É também uma fase de preparação física e psicológica, para o nascimento e para a parentalidade (Coutinho et al, 2014)

O pré-natal é realizado de forma multidisciplinar, cujo objetivo será a manutenção da integridade das condições de higiene fetal e cujos resultados devem ser avaliados em longo prazo, com a formação de elementos físicos e intelectualmente úteis à comunidade e ao país. As consultas mensais, quinzenais conforme necessidade e acontecem intercaladas pela enfermagem e medicina. Durante as consultas, pesquisam- -se afecções orgânicas e distúrbios emocionais, orienta-se o preparo psicológico para o parto e instituem-se normas de higiene-dietéticas, realiza-se o preparo psicológico para o parto e inicia-se o tratamento para eventuais doenças intercorrentes (Silva, 2013).

A assistência multiprofissional pode colaborar nas ações de pré-natal, sendo os profissionais da equipe da Estratégia Saúde da Família (ESF), da equipe da Estratégia Saúde da Família-Saúde Bucal (ESF-SB), do Núcleo de Apoio à Saúde da Família (NASF) e saúde mental, utilizando-se das várias modalidades de prática clínica úteis para o cuidado do usuário com condição crônica (Saúde, 2020)

O Brasil apresenta aumento na proporção de gestantes com pelo menos sete consultas de pré-natal e parto assistidas por profissionais de saúde, sendo necessário o aprimoramento da qualidade dessas consultas e o cuidado no parto e pós-parto (Tomazi et al, 2019).

\subsection{Parto e nascimento}

Gravidez e parto são eventos sociais que compõem a experiência de reprodução humana, tanto do homem como da mulher. Sendo uma vivência acompanhada de amadurecimento, tanto mental quanto físico, e que apresenta também aos pais situações singulares, dentre eles o cuidado integral na garantia do bem estar de outro ser totalmente dependente. O nascer traz mudanças não somente aos pais, mas a família de forma geral, e até mesmo a nível de comunidade, sendo os pais coadjuvantes na vida de uma nova pessoa (Strapasson; Nedel, 2010).

A gestante tem direito ao plano de Parto, podendo elaborar o plano durante todo o pré-natal, tendo tempo para esclarecer dúvidas, dialogar com os profissionais e ouvir a experiência de outras mulheres (Saúde, 2020).

Dessa forma, a presença dos parceiros durante o parto e puerpério é extremamente importante, influenciando até mesmo no desenvolvimento da criança e garantindo autonomia ao casal diante de possíveis situações de emergência, já as 
mulheres solteiras apresentam uma maior dependência da equipe de saúde, buscando-a de forma rotineira diante das dificuldades, podendo apresentar riscos na constituição da maternidade (Saúde, 2002).

\subsection{Puerpério}

O puerpério, comumente denominado resguardo ou pós-parto é definido como o período em que ocorrem as alterações no corpo feminino para que este volte a ser como era antes da gestação, havendo realocação dos órgãos, alterações físicas e psicológicas nas mulheres que vivem essa fase. Esse período tem início no momento da expulsão do feto e da placenta e é marcado pela, especialmente, pela obtenção da identidade materna, onde a mulher aprender a ser mãe, adaptando-se ao novo contexto de cuidadora e de vínculo entre a família com o novo ser que chega (Oliveira et al, 2012).

Em tal contexto, puerpério tem definição clara de um período do ciclo gravídico puerperal, onde acontecem alterações clássicas que são acarretadas pelos eventos: gravidez e parto, no corpo da mulher. O pós-parto pode ser caracterizado por sentimentos à flor da pele, como parto, onde se espera o nascimento de um filho saudável - abrindo um leque de autoconfiança; desânimo físico - em relação ao tipo de parto ao qual foi submetida; sentimento de decepção - com o corpo ou aparência física (Strapasson \& Nedel, 2010)

O puerpério é dividido, basicamente, em três fases, sendo elas: a imediata, que vai do $1^{\circ}$ ao $10^{\circ}$ dia, o tardio que compreende o período entre o $11^{\circ}$ ao $45^{\circ}$, e o remoto que do $45^{\circ}$ em diante, observando que esse período só termina quando a mulher tem suas funções reprodutivas reestabelecidas, ou seja, volta a ovular (Vieira et al., 2010).

Ao final do período puerperal, a mulher deve ser orientada com relação à continuidade do cuidado com sua saúde, no planejamento familiar, no acompanhamento das condições crônicas diagnosticadas durante a gestação e para o fortalecimento da capacidade de autocuidado (Saúde, 2020).

\subsubsection{Complicações ocorrentes no puerpério}

Por se tratar de um processo fisiológico traumático o parto pode acometer complicações que perduram durante o período de puerpério e, portanto, é necessário um olhar atento dos profissionais da saúde para os sinais e sintomas que venham a surgir no momento do parto e nos dias que compreendem o puerpério.

A cesariana vem sendo uma prática cada vez mais comum, devido a sua praticidade é a via de parto de preferências da maioria dos obstetras, e também de algumas gestantes que romantizam o parto programado, contudo essa intervenção é subestimada quanto às suas complicações, tanto para a mãe quanto para o bebê. Zimmerman et al, 2009 define como as principais complicações relacionadas a cesariana os acidentes anestésicos, hemorragias, lesões viscerais e intestinais, alargamento da invasão miometrial, embolia amniótica e infecção puerperal. Há ainda uma maior incidência de endometrite puerperal nos casos de parto por cesariana.

A incidência de infecção puerperal é significativa e está relacionada como uma das principais causas de morbimortalidade no pós-parto, no Brasil está em quarto lugar como causa de mortalidade materna (Machado; Praça, 2005).

As complicações mamárias compõem outro conjunto de problemas de saúde que pode acometer as puérperas, sendo desencadeados pela falta de informação e dos cuidados com a lactação. As complicações mais comuns nesse âmbito são as mastites, que podem acometer um ou ambas as mamas, as fissuras mamilares, que podem ocorrem também em uma ou ambas as mamas e podem ser pequenas, média euforia e alívio; frustrações acerca da expectativa ou grandes, ou na ainda vesicular e o ingurgitamento mamilar que é caracterizado pela mama "empedrada" (Skupien et al, 2016). 


\subsection{Assistência de enfermagem no puerpério}

É importante salientar que, ao se tratar da saúde da mulher, a assistência materna vive uma busca por melhorias, especialmente no combate a mortes de mulheres em idades reprodutivas, decorrente de complicações no parto e puerpério. Segundo Serruya 2004, esses cuidados, no Brasil, ainda são centrados em uma assistência voltada para medicação, hospitalização e tudo de forma mecanizada, sem um olhar holístico.

Diante da perspectiva do cuidado na atenção primária a assistência de enfermagem na saúde da mulher ocorre de forma efetiva, visto que o profissional e a paciente têm um contato prolongado, que vem antes da gestação com cuidados do profissional de enfermagem, que participa ativamente do pré-natal, com consultas mensais e oferta de educação em saúde abordando temas voltados para as gestantes. Esse cuidado perdura por todo o puerpério, onde são domiciliares a mãe e ao bebê, para acompanhar a evolução de ambos, sendo que na puérpera a busca é por identificar as alterações fisiológicas, a capacidade de cuidado de si e do bebe e também as possíveis complicações que possam surgir (Vieira et al., 2011).

Ainda é possível observar uma falta de capacitação dos profissionais em relação aos diagnósticos de enfermagem para puérperas e ainda uma abordagem insatisfatória quanto a orientação dos cuidados a serem realizados. É possível observar que mesmo os profissionais passam informações e têm condutas baseadas em crenças e culturas, sem nenhum embasamento científico (Vieira et al., 2010; Gomes et al., 2020).

\subsection{Diagnósticos de enfermagem no período do puerpério}

Sabe-se que o período do puerpério demanda cuidados específicos e que devem ser aplicados de acordo com as necessidades de cada paciente, assim se faz necessário a aplicação de diagnósticos de enfermagem, que variam de acordo com o do período e respeitando as mudanças psicobiologicas das pacientes (Lemos et al, 2012; Oliveira et al, 2021).

No estudo realizado por Vieira 2011, foram abordados os diagnósticos de enfermagem pertinentes aos períodos imediato e tardio do puerpério, sendo aplicados, de acordo com o NANDA, diagnósticos relacionados a conceitos culturais das pacientes e a informações passadas por profissionais da saúde, sendo alguns deles:

- Conhecimento deficiente;

- Armazenamento de leite materno;

- Cuidados prestados a sua criança;

- Amamentação

- Cuidados com as mamas

- Cuidados com incisão cirúrgica abdominal ou perineal ou laceração;

- Risco de amamentação interrompida ou ineficaz;

- Risco de infecção.

Os diagnósticos relacionados à amamentação devem ser feitos considerando, entre outras coisas a relação entre mãe e filho, a comunicação e a verbalização, e ainda observar os sinais de realizadas as consultas puerperais satisfação do bebê, presença de eliminações e suas características e ainda a procura do bebê pelo alimento e sua capacidade de sugar e deglutir o leite (Vieira et al., 2011).

Para Patine 2006, quanto aos diagnósticos atribuídos a pacientes puérperas em alojamento conjunto, o se encontram as puérperas em fase imediata, podem ser além dos já citados acima:

- Dor aguda, relacionada ao processo de parto e necessidade de intervenção cirúrgica ou pontos em episiotomias e lacerações;

- Risco de constipação;

- Ansiedade, diante da nova rotina e do processo de adaptação mãe e filho; 
- Padrão de sono prejudicado, devido está alocado em um local com outras pessoas e também aos cuidados em tempo integral destinados ao bebê;

- Desempenho de papel ineficaz, especialmente para mães de primeira viagem que não tem conhecimento prático sobre os cuidados com ela e com o filho;

- Mobilidade física prejudicada, as pacientes que passaram por cesariana e precisam adaptar-se a locomoção com os pontos.

A importância é de que os enfermeiros saibam reconhecer as necessidades dos pais e dos familiares no período do puerpério, para que possam prestar os cuidados de forma mais específica, respeitando as necessidades que esse período demanda, para tanto é preciso que sejam definidos os diagnósticos de enfermagem e planejadas as intervenções que cada um objetiva (Patine; Furlan, 2006; Silva et al, 2021).

\section{Metodologia}

Trata-se de um estudo secundário, uma vez que estabelece conclusões a partir de estudos primários, que já foram publicados na literatura. Foi realizada uma revisão de literatura do tipo descritiva com abordagem narrativa. As publicações desse estilo são amplas, apropriadas para descrever e discutir o desenvolvimento ou o "estado da arte" em relação a perspectiva de um assunto, sob ponto de vista teórico ou contextual (Rother, 2007; Schwartz et al 2020).

Realizou-se a busca por artigos publicados em bancos de pesquisa como Bireme, SCielo e PubMed. Utilizou-se como palavras chaves e critérios de inclusão as palavras: "Cuidados de Enfermagem", "Puerpério", "Saúde da Mulher" associada ao marcador booleano AND.

Como critério de exclusão de artigos, foram ignorados os que não eram tidos como fundamentais para a escrita do artigo, sendo estes, artigos publicados anteriormente ao ano 2000, artigos publicados em outro idioma que não seja o português, inglês, espanhol ou não possuam tradução para tal, materiais incompletos ou que não atendam ao conteúdo central do estudo. Utilizou-se uma abordagem qualitativa que se atém apenas ao conteúdo do de interesse da pesquisa.

\section{Resultados e Discussões}

Realizou-se uma pesquisa em banco de dados como o Scielo, Pubmed e Bireme. Selecionaram-se 10 artigos científicos, disponíveis nas bases de dados informadas acima, que melhor atendiam às necessidades da pesquisa atendendo aos critérios de inclusão e exclusão.

Foram utilizados 10 artigos que atendiam a temática de forma ampla e integral, abordando tópicos como: interação entre enfermeiros e puérperas; importância e realização de consultas puerperais; saúde da mulher; complicações ocorrentes no puerpério e percepção das puérperas sobre seu estado momentâneo, conforme exposto no Quadro 1. 
Quadro 1: Quadro sinóptico da base de dados Scielo.

\begin{tabular}{|c|c|c|c|c|}
\hline $\begin{array}{c}\text { Base } \\
\text { de } \\
\text { dados }\end{array}$ & Título & Autor & Ano & Metodologia \\
\hline Scielo & $\begin{array}{l}\text { Interação Enfermeiros e } \\
\text { Puérperas: na procura de um } \\
\text { cuidado cultural. }\end{array}$ & Coutinho et al.. & 2019 & $\begin{array}{c}\text { Estudo qualitativo. Na coleta de dados se } \\
\text { utilizou a entrevista semiestruturada, a } 21 \\
\text { enfermeiras e } 15 \text { puérperas num total de } 36 \\
\text { entrevistadas. }\end{array}$ \\
\hline Scielo & $\begin{array}{c}\text { Frequência e fatores } \\
\text { associados à não realização } \\
\text { da consulta puerperal em um } \\
\text { estudo de coorte. }\end{array}$ & Gonçalves et al. & 2019 & $\begin{array}{l}\text { Estudo de coorte prospectiva da coleta de dados } \\
\text { em dois momentos, nas primeiras } 48 \mathrm{~h} \text { e após o } \\
42^{\circ} \text { dia pós-parto. }\end{array}$ \\
\hline Scielo & $\begin{array}{c}\text { Saúde da mulher na } \\
\text { gestação, parto e puerpério: } \\
25 \text { anos de recomendações } \\
\text { de organismos } \\
\text { internacionais. }\end{array}$ & Parada et al & 2019 & Editorial. \\
\hline Scielo & $\begin{array}{c}\text { Sintomas depressivos } \\
\text { maternos no puerpério } \\
\text { imediato:fatores associados }\end{array}$ & Poles et al. & 2018 & $\begin{array}{c}\text { Estudo transversal, realizado com } 1099 \\
\text { puérperas. }\end{array}$ \\
\hline Scielo & $\begin{array}{l}\text { Percepções da puérpera de } \\
\text { alto risco acerca do } \\
\text { ambiente hospitalar à luz de } \\
\text { Florence Nightingale. }\end{array}$ & Roque et al. & 2015 & $\begin{array}{l}\text { Estudo narrativo com abordagem } \\
\text { qualitativa,sustentado em Florence Nightingale, } \\
\text { realizado em uma instituição pública do sul } \\
\text { do Brasil, de janeiro à março de } 2010 \text {. }\end{array}$ \\
\hline Bireme & $\begin{array}{c}\text { Diagnóstico de Enfermagem } \\
\text { Relacionados ao Alojamento } \\
\text { Conjunto Revista de } \\
\text { Enfermagem }\end{array}$ & Martins et al. & 2021 & $\begin{array}{l}\text { É estudo bibliográfico, descritivo, tipo revisão } \\
\text { integrativa, com busca nas bases de dados: } \\
\text { lilacs, medline e na biblioteca virtual scielo de } \\
2010 \text { a } 2020 .\end{array}$ \\
\hline Bireme & $\begin{array}{c}\text { Satisfação da gestante com a } \\
\text { assistência ao parto e pós- } \\
\text { parto e variáveis associadas }\end{array}$ & Arrebola et al. & 2021 & $\begin{array}{c}\text { Trata-se de um estudo longitudinal, } \\
\text { observacional. Realizada análise descritiva e } \\
\text { inferencial. }\end{array}$ \\
\hline Bireme & $\begin{array}{c}\text { Assistência ao puerpério e a } \\
\text { construção de um } \\
\text { fluxograma para consulta de } \\
\text { enfermagem }\end{array}$ & Silva et al. & 2020 & $\begin{array}{l}\text { Realizado estudo exploratório descritivo, } \\
\text { transversal, em um hospital privado no Brasil, } \\
\text { com amostra de } 114 \text { mulheres. }\end{array}$ \\
\hline Pubmed & $\begin{array}{l}\text { Prática de enfermagem na } \\
\text { atenção domiciliar: uma } \\
\text { revisão integrativa da } \\
\text { literatura. }\end{array}$ & Andrade et al. & 2017 & $\begin{array}{l}\text { Trata-se de uma revisão integrativa nas bases de } \\
\text { dados lilacs, bdenf, ibecs e medline. }\end{array}$ \\
\hline
\end{tabular}

Fonte: Pesquisadores (2020).

Parada, (2019) abordou em seu estudo a saúde da mulher, de forma ampla a acerca da gravidez, parto e puerpério, evidenciando que a temática vem sendo cada vez mais discutida devido às complicações desses períodos que acarretam nas altas taxas de óbitos de mulheres em idades reprodutivas. Para Costa 2009, ao longo dos anos as mulheres foram conquistando de forma autônoma sobre o próprio corpo e também direito a escolhas sobre o planejamento familiar. Contudo estas mulheres não tiveram uma educação que acompanhasse essas conquistas, e foi nesse momento que a saúde da mulher se tornou um problema de saúde pública ao considerar que a morte por complicações na gestação ou puerpério está entre as principais causas de mortes de mulheres em idade fértil.

Segundo Gonçalves 2019, voltou seu estudo para as causas relacionadas a ausência de consultas puerperais, objetivando evidenciar a importância da revisão puerperal para identificar fatores de riscos à saúde da mãe e do bebê. Neste estudo observou-se que quase $25 \%$ das puérperas não realizam a revisão pós-parto. Já para Neves 2021, há uma satisfação em "logística e meio ambiente" e em "atendimento ao parto" (99,0\% e 97,6\% respectivamente); e observa-se que os aspectos com 
os quais as gestantes apresentaram maior grau de insatisfação ou indiferença foram os cuidados com o recém-nascido (12,3\%) e o puerpério $(7,9 \%)$.

Assim é preciso que as complicações puerperais e os fatores de riscos a vidas da mãe e do bebê sejam lembrados com a mesma ênfase que os riscos relacionados ao parto e os cuidados empregados a gestação, não de forma terrorista, mas com a elaboração e implantação de políticas em saúde acerca do tema (Cavalgante et al 2021).

Segundo, Martins et al (2021), consideraram em seu estudo fatores de elegibilidade para a mãe e o filho serem encaminhados ao alojamento conjunto: puérperas estáveis e sem contraindicações para a permanência junto ao RN.

Para Poles (2018), dentre as complicações comuns no puerpério a depressão pós-parto é uma das mais frequentes, abordando em seu estudo os sintomas e os fatores de risco para que esta ocorra. Foi possível identificar que quase $7 \%$ das entrevistadas apresentaram estado de depressão, e os fatores mais associados foram, uso de antidepressivos na gestação, violência doméstica e parto por cesariana. Assim fica exposto que por mais que o estado depressivo seja ocorrente no puerpério, ele pode ser iniciado antes mesmo da gestação, havendo assim a necessidade de um olhar mais humano para essas pacientes que apresentem fatores de risco.

Para Silva (2020), quanto às características do nascimento, houve predomínio da prática de alojamento conjunto e aleitamento materno exclusivo. Quanto ao exame físico, há alterações sugestivas de infecção de ferida operatória em 17 $(14,9 \%)$ mulheres e alterações mamárias em cinco $(4,4 \%)$. No estudo, constatou-se também que oito $(7,0 \%)$ mulheres necessitam receber reforço quanto às orientações sobre como fazer a ordenha manual, e duas (1. 8\%) mulheres estavam emocionalmente instáveis, pois relataram dificuldades na alimentação devido à perda abrupta de apetite e dificuldades para dormir mesmo quando o bebê estava dormindo.

Por outro lado, Poles et al (2021), o ponto é que ocorra uma maior capacitação dos profissionais sobre o direito de escolha das pacientes, especialmente relacionados a via de parto, pois quando a mulher engravida ela passa por um processo de construção para o parto e este é extremamente romantizado por ela, e qualquer coisa que saia fora do planejado lhe causará frustração.

Já Roque e Carraro, (2015), abordaram um tema semelhante ao de Gonçalves, contudo voltou-se para as puérperas de alto risco e tentaram determinar a percepção delas sobre o ambiente hospitalar e os cuidados destinados a elas. Neste estudo foram incluídas mulheres que passaram por parto prematuro e internação neonatal e o que essas situações influenciaram no seu modo de visão.

É preciso que se tenha um olhar mais cuidadoso e empático para as puérperas de alto risco, visto que além do próprio sofrimento elas enfrentam também o sofrimento dos filhos que passam por complicações neonatais e necessitam de internações. Nesse momento a enfermagem deve servir como um ponto de apoio, informando, consolando e dando assistência em tudo o mais que for necessário. Nessa situação, o enfermeiro deve se atentar a fornecer informações sobre estado de saúde do bebê, sobre o ambiente hospitalar, ambiente de internação neonatal e mostra-se prestativo diante de qualquer necessidade (Parada, 2019).

Como exposto no quadro acima, Coutinho (2019) realizou estudo tendo por objetivo identificar o grau de interação entre enfermeiros e puérperas, onde ambas participaram evidenciando seus pontos de vista sobre o cuidado a pacientes no pósparto. Para as puérperas a característica que mais se destaca é as formas e disponibilidade de cuidados, onde as pacientes caracterizam as profissionais como competentes, atenciosas, simpáticas e capacitadas, dentre outras qualidades. Já para Andrade (2017), a grande questão é a atuação do enfermeiro na atenção domiciliar, que apresenta complexidade e diversidade de ações, com uso de tecnologias leves, leve-duras (principalmente) e duras.

Segundo Gonçalves (2019), dentre outros importantes influenciadores as pacientes destacaram o processo e a forma de cuidar, mostrando que antes de qualquer coisa a enfermagem é caracterizada pela forma como interage com os pacientes 
durante as assistências, o da realização do pré-natal, acreditam que está significativamente vinculada a enfermagem, sendo esta considerada a arte do cuidar. As puérperas também relatam como ponto positivo as formas de comunicação, os meios utilizados para sanar dúvidas e realizar a educação em saúde como ponto positivo, e ainda o respeito por suas crenças e culturas no processo de cuidado, tanto no autocuidado como nos cuidados com o bebê. O que evidencia que a enfermagem tenta passar as formas corretas de cuidados de forma natural, sem desrespeitar as vontades das pacientes ou criticar sua visão de cuidado.

Andrade et al (2017), evidencia em seu estudo que os enfermeiros são essenciais na atenção domiciliar, tanto pelo conhecimento específico do tratamento terapêutico quanto por estarem na vanguarda ao ensinar aos pacientes e familiares os cuidados necessários, por exemplo, ao manusear os equipamentos com segurança e eficiência. Nesse sentido, Parada (2019), demonstra que a enfermagem tem um papel importante na implantação das políticas de saúde voltadas para mulheres, especialmente porque os profissionais que atuam na atenção primária têm um contato mais íntimo com seus pacientes, e é a primeira escolha para passar informações e sanar dúvidas.

\section{Considerações}

Ao entender que o puerpério não é somente um período em que a mulher se adapta a chegada do filho, compreende-se também que esse momento demanda cuidados mais específicos, onde a mulher deve ser vista de forma integral tendo suas necessidades atendidas.

A enfermagem deve atuar com um olhar mais holístico, integralizado, tentando trazer para dentro dos cuidados não só a paciente, mas também tudo o que está a sua volta, as condições sociais, econômicas, psicológicas e qualquer outra que possa causar alguma espécie de frustração à puérpera. Para tanto é preciso que os profissionais passem por um processo de desconstrução sobre os cuidados a serem prestados a puérperas e que possam respeitar o seu direito à autonomia na assistência.

É necessária uma busca pelo saber sobre ações reproduzidas para assistência ao Puerpério pelos profissionais de saúde, principalmente aos enfermeiros, que auxiliam a descontruir barreiras, aproximar diálogos e contribuir para uma assistência mais integralizada. Estudos nessa área podem contribuir para aprimorar as políticas públicas e fortalecer diálogos no meio acadêmico.

\section{Referências}

Baker, D; (2020). minhavontade, UNFPA. https://popdesenvolvimento.org/images/noticias/SWOP_2020_relatorio_PT.pdf.

Brasil,. (1996). “L9263." Planalto 1996, http://www.planalto.gov.br/ccivil_03/leis/19263.htm.

Bouzas, I.; Monteiro, C. (2010). Alteração do ciclo menstrual na adolescência: manifestação precoce da Síndrome Metabólica? Universidade do Estado do Rio de Janeiro 2010, https://www.bdtd.uerj.br:8443/handle/1/8700.

Brasil. (2006). Atenção à saúde da mulher no Pré-Natal, Puerpério e Cuidados ao Recém-nascido. Secretaria de Saúde do Distrito Federal, https://www.saude.df.gov.br/wp-conteudo/uploads/2018/04/3 Atencao_a_Saude_da_Mulher_no_Prenatal_Puerperio_e_Cuidados_ao_Recem_nascido.pdf.

Brenes, A. (1991). História da parturição no Brasil, século XIX. Cadernos de Saúde Pública. https://www.scielo.br/j/csp/a/xFmLWvbx9BRGyJXW38gFXpP/?lang=pt.

Cardosoa, Edméia, and Coelho. "Enfermeiras que cuidam de mulheres: conhecendo a prática sob o olhar de gênero | São Paulo; sn; dez. 2001. 174f p. | BDENF.” Pesquisa .bvsalud .org, 2001, https://pesquisa.bvsalud.org/portal/resource/pt/bde-17743.

Carvalho, G., et al. (2007). A idade da menarca está diminuindo?” Redalyc, http://www.redalyc.org/pdf/4060/406038920014.pdf.

Cavalcante, L. G. ., Barbosa, D. A. ., Carvalho , B. B. de ., Souza, J. T. A. H. de ., Oliveira, R. T. S. ., Costa, G. F. C., Lima, T. O. S., Silva, R. A. N. ., Santana, M. D. O. . ., \& Abrão, R. K. . (2021). Estratégias do enfermeiro obstetra para diminuição dos métodos intervencionistas durante o parto normal. Research, Society and Development, 10(2), e49510211896. https://doi.org/10.33448/rsd-v10i2.11896

Coelho, E. et al. (2009). Integralidade do cuidado à saúde da mulher: limites da prática profissional. Esc. Anna Nery. https://www.scielo.br/scielo.php?pid=S1414-81452009000100021\&script=sci_abstract\&tlng=pt. 
Corrêa, M. et al. (2017). Acolhimento no cuidado à saúde da mulher no puerpério.” Cadernos de Saúde Pública 2017 , https://www.scielo.br/j/csp/a/GbrsTdSmBsXcLSF6JPH6QJD/?lang=pt.

Costa, M; , et al. (2015). Episiotomia no Parto Normal: Incidência e Complicações | Carpe Diem: Revista Cultural e Científica do Unifacex.” Centro Universitário Facex - Unifacex, 18 December, https://periodicos.unifacex.com.br/Revista/article/view/655.

Costa, A. (2009). Participação social na conquista das políticas de saúde para mulheres no Brasil.Ciência \& Saúde Coletiva. https://www.scielo.br/j/csc/a/x7HVb8dmB9wRHNC3JgjT6yQ/?lang=pt.

Coutinho, E., et al. (2014). Nurses- puerperal mothers interaction: searching for cultural care. Revista Brasileira de Enfermagem - Nurses- puerperal mothers interaction: searching for cultural care Nurses- puerperal mothers interaction: searching for cultural care, https://www.scielo.br/j/reben/a/hyC7Hxf7htw34LnbcXLT73p/?lang=pt.

Coutinho, E. et al. (2019). Gravidez e parto: O que muda no estilo de vida das mulheres que se tornam mães? Ao.” SciELO, https://www.scielo.br/j/reeusp/a/sHRmhNMCs4j77gZvbYxRydC/?lang=pt\&format=pdf.

Durval, S. et al. (2016). Cuidado de enfermagem na assistência à saúde da mulher no climatério. Revista Saúde; http://revistas.ung.br/index.php/saude/article/view/2644.

Gomes, A. V. ., Ferreira , R. K. A. ., \& Rodrigues, C. F. do C. . (2020). A saúde na vida do cárcere no Brasil e no Tocantins . Research, Society and Development, 9(9), e981998067. https://doi.org/10.33448/rsd-v9i9.8067

Gonçalves, C., et al. (2019). Frequency and associated factors with failure to perform the puerperal consultation in a cohort study. Revista Brasileira de Saúde Materno Infantil-, https://www.scielo.br/j/rbsmi/a/7H57NvDHHzYD8xVRBhQqBnD/?lang=pt.

Jorge, H, et al. (2015). Assistência pré-natal e políticas públicas de saúde da mulher: revisão integrativa | Revista Brasileira em Promoção da Saúde.” Portal de Periódicos da Universidade de Fortaleza, 30 March, https://periodicos.unifor.br/RBPS/article/view/2864.

Justino, G., et al. (2019). Saúde sexual e reprodutiva no puerpério: vivências de mulheres | Rev. enferm. UFPE on line;13: 1-10. https://pesquisa.bvsalud.org/portal/resource/pt/biblio-1046593.

Lemos, Renata, et al. (2012). Diagnósticos de Enfermagem identificados durante o período puerperal imediato: estudo descritivo. Revista de Enfermagem do Centro-Oeste Mineiro. http://www.seer.ufsj.edu.br/index.php/recom/article/view/183.

Machado, N.; Neide P. (2005). Infecção puerperal em Centro de Parto Normal: ocorrência e fatores predisponentes, Reben. https://www.scielo.br/j/reben/a/s9483HdJB3sdpj47xLzcb7C/abstract/?lang=pt.

Marques, D:, Adriana P.. (2011). Assistência pautada nos direitos sexuais e reprodutivos: uma condição para promover a saúde da mulher. Revista Eletrônica de Enfermagem. 30 September.,https://revistas.ufg.br/fen/article/view/9668.

Martins, A., et al. (2021) Diagnóstico de Enfermagem Relacionados ao Alojamento Conjunto. Revista de Enfermagem UFPE on line. https://periodicos.ufpe.br/revistas/revistaenfermagem/article/view/245163/37735.

Monk, F. (2011). Redalyc.Ciclo menstrual: sintomatología y regularidad del estilo de vida diario. Redalyc. https://www.redalyc.org/pdf/184/18426920004.pdf. Accessed 14 January 2020.

Medeiros, L.; Costa, A.C.. (2016). Período puerperal: a importância da visita domiciliar para enfermeiros da Atenção Primária à Saúde.” Redalyc, 2016, https://www.redalyc.org/pdf/3240/324044160015.pdf.

Navas, R., et al. "Women's satisfaction with childbirth and postpartum care and associated variables. Revista da Escola de Enfermagem da USP 2021, https://www.scielo.br/j/reeusp/a/dfnMzVQpwNDvCj54thCxh6L/?lang=en.

Nunes, E. (2007). O desafio do conhecimento: pesquisa qualitativa em saúde. Ciência \& Saúde Coletiva https://www.scielo.br/j/csc/a/FgpDFKSpjsybVGMj4QK6Ssv/?lang=pt.

Oliveira, J., et al. (2016). Padrão hormonal feminino: menopausa e terapia de reposição. Revista Rbac, http://www.rbac.org.br/artigos/padrao-hormonalfeminino-menopausa-e-terapia-de-reposicao-48n-3/. Accessed 14 January 2020.

Oliveira, L. L. S. de, Lima, T. O. S. ., Silva, R. A. N., Silva, R. M. O. ., Abreu, V. P. L. ., \& Ferreira, R. K. A. . (2020). Atuação do enfermeiro na assistência a mulher com câncer de ovário . Research, Society and Development, 9(9), e43996962. https://doi.org/10.33448/rsd-v9i9.6962

Oliveira, J. et al. (2016). Percepção das puérperas quanto aos cuidados prestados pela equipe de saúde no puerpério. Rev Rene, http://www.periodicos.ufc.br/rene/article/view/3772.

Parada, C. (2019). Women's health during pregnancy, childbirth and puerperium: 25 years of recommendations from international organizations. Revista Brasileira de Enfermagem, https://www.scielo.br/j/reben/a/w9NjtPShP8F7Yfp9ShXbZDh/?lang=pt.

Patine, F.; et al (2006). Diagnósticos de enfermagem no atendimento a puérperas e recém-nascidos internados em alojamento conjunto; Bireme. http://bases.bireme.br/cgi-

bin/wxislind.exe/iah/online/?IsisScript=iah/iah.xis\&src=google\&base=LILACS\&lang=p\&nextAction=lnk\&exprSearch=485865\&indexSearch=ID

Poles, M., et al. (2018) Sintomas depressivos maternos no puerpério imediato: fatores associados. Acta Paulista de Enfermagem - Brasil- - Sintomas depressivos maternos no puerpério imediato: fatores associados, https://www.scielo.br/j/ape/a/HMjZg8HJgbMdsJysnyQsYjL/?lang=pt.

Riul, Sueli, et al. (2018). Ações Educativas na Área da Saúde da Mulher - Relato de Experiência de Extensão Universitária -Revistas Eletrônicas - UFTM, 7 August, 
https://seer.uftm.edu.br/revistaeletronica/index.php/enfer/user/setLocale/es_ES?source=\%2Frevistaeletronica\%2Findex.php $\% 2$ Fenfer $\% 2$ Farticle $\% 2$ Fview $\% 2 \mathrm{~F}$ $2302 \% 2 \mathrm{~F} 0$

Rocha, C. C., Lima, T. S., Silva, R. A. N., \& Abrão, R. K. (2020). Abordagens sobre sífilis congênita . Research, Society and Development, 9(8), e984986820. https://doi.org/10.33448/rsd-v9i8.6820

Rodrigues, C. F. do C. ., Alves, M. M. M. ., Brustulin, R. ., \& Ferreira, R. K. A. . (2020). Avaliação do Controle do HIV/Aids na Atenção Primária em Palmas/TO. Research, Society and Development, 9(9), e372997126. https://doi.org/10.33448/rsd-v9i9.7126

Rodrigues, C. F. do C. ., Silva, M. da V. F. B. ., Souto, L. F. de S. ., Silva, E. A. A. da ., Mocelai, R. S. ., Rodrigues, A. L. M. ., Coelho, S. C. D. ., \& Abrão , R. K. . (2020). Promoção de saúde para mulheres em território de vulnerabilidade social: comunidade a Saroba. Research, Society and Development, 9(10), e8159109116. https://doi.org/10.33448/rsd-v9i10.9116

Roque, A., and Telma C. (2015). Perceptions about the hospital environment from the perspective of high-risk puerperal women based on Florence Nightingale's theory. Revista Gaúcha de Enfermagem, https://www.scielo.br/j/rgenf/a/YzGQrMMq6VYWCcTrkmkvLxy/?lang=pt.

Rother, E. “Brasil - Revisão sistemática X revisão narrativa Revisão sistemática X revisão narrativa.” SciELO, 2007, https://www.scielo.br/j/ape/a/z7zZ4Z4GwYV6FR7S9FHTByr/?lang=pt.

Santiago, L. et al. "Direitos da Mulher Trabalhadora na Gravidez no Pós-Parto e Durante o Aleitamento Materno.” Sociedade Brasileira de Pediatria, 2012, https://www.sbp.com.br/fileadmin/user_upload/2012/12/Direitos-da-Mulher-Trabalhadora-na-Gravidez-no-Ps-Parto-e-Durante-o-Aleitamento-Materno.pdf.

Santos., M., et al. Rezende Obstetrícia Rezende Obstetrics Rezende Obstetricia Resenha de Livro, https://periodicos.ufpe.br/revistas/revistaenfermagem/article/download/237726/30843.

Santos, T. et al. "Fatores que Contribuem para o Início da Atividade Sexual em Adolescentes: Revisão Integrativa | Revista de Atenção à Saúde. 6 July 2015, https://seer.uscs.edu.br/index.php/revista_ciencias_saude/article/view/2668.

Santos, J. C. M. dos ., Silva , T. R. de S. ., Aragão, M. A. M. ., Abreu, V. P. L. ., Silva , R. A. N. ., Santana , M. D. O. ., \& Abrão , R. K. . (2020). A Percepção das puérperas sobre o parto vaginal humanizado assistido pela equipe de Enfermagem. Research, Society and Development, 9(10), e5459108361. https://doi.org/10.33448/rsd-v9i10.8361

Saúde (2012). Untitled, http://189.28.128.100/dab/docs/publicacoes/geral/pnab.pdf.

Saúde (2006). Política Nacional de Atenção Integral à Saúde da Mulher. Conselho Nacional de Saúde. https://conselho.saude.gov.br/ultimas_noticias/2007/politica_mulher.pdf.

Saúde. (2008). Manual de Atenção à Mulher no Climatério / Menopausa. Biblioteca Virtual em Saúde, 24 July; https://bvsms.saude.gov.br/bvs/publicacoes/manual_atencao_mulher_climaterio.pdf.

Saúde. (2020). Saúde da Mulher na Gestação, Parto e Puerpério. Atenção Básica do RS,.

https://atencaobasica.saude.rs.gov.br/upload/arquivos/202001/03091259-nt-gestante-planificasus.pdf.

Schwartz, S., Vieira, M. A., Rodrigues, A. C. S., \& Ferreira, R. K. A. (2020). Estratégias para o trabalho com textos na universidade. Research, Society and Development, 9(8), e790986209. https://doi.org/10.33448/rsd-v9i8.6209

Serruya, S., et al. (2004). O Programa de Humanização no Pré-natal e Nascimento do Ministério da Saúde no Brasil: resultados iniciais. Cadernos de Saúde Pública, https://www.scielo.br/j/csp/a/9nJfmGQRd3jYxMRNkHRJKpH/abstract/?lang=pt.

Silva, E. (2013). Gestação e preparo para o parto: programas de intervenção. Centro Universitário São Camilo. https://bvsms.saude.gov.br/bvs/artigos/mundo_saude/gestacao_preparo_parto_programas_intervencao.pdf.

Silva, L., et al. (2020). Assistance to the puerperium and the construction of a flow chart for nursing consultation. Revista Brasileira de Saúde Materno Infantil, https://www.scielo.br/j/rbsmi/a/jjsBnwhpS4K5FT4WMn8zH7d/?lang=en.

Silva, T. R. de S. ., Santos, J. C. M. dos ., Oliveira, J. S. de ., Abreu, V. P. L. ., Silva, R. R. da ., Dantas, K. L. S. ., Silva, R. M. O. ., Januário, P. O. da S. ., Gonçalves, G. F. de S. ., Rodrigues, C. F. do C. ., Silva, M. da V. F. B. ., Oliveira, R. T. S. ., \& Ferreira , R. K. A. (2021). A importância do exame Preventivo de Câncer de Colo de Útero e os fatores relacionados a não adesão . Research, Society and Development, 10(4), e51710414079. https://doi.org/10.33448/rsdv10i4.14079

Skupien, S., et al. (2016). Consulta Puerperal de Enfermagem: Prevenção de Complicações Mamárias | Skupien | Cogitare Enfermagem. Biblioteca Digital de Periódicos, Redalyc, https://revistas.ufpr.br/cogitare/article/view/44691.

Strapasson, M., and Maria N. (2010). Puerpério imediato: desvendando o significado da maternidade. SciELO, Revista Gaúcha de Enfermagem , https://www.scielo.br/j/rgenf/a/KQydgDyHVrKHWMQDfTDmfFJ/abstract/?lang=pt.

Teixeira, A. (2012). Efeito das diferentes fases do ciclo menstrual na composição corporal de universitárias. REDIB https://redib.org/Record/oai_articulo610371-efeito-das-diferentes-fases-do-ciclo-menstrual-na-composi\%C3\%A7\%C3\%A3o-corporal-deuniversit\%C3\%A1rias.

Tomasi, Y. et al. (2019). Do pré-natal ao parto: um estudo transversal sobre a influência do acompanhante nas boas práticas obstétricas no Sistema Único de Saúde em Santa Catarina. SciELO, https://www.scielo.br/j/ress/a/ZHFXkKHPPypjwbtHCxsRjqP/?lang=pt.

Valença, C. et al. (2010). Mulher no climatério: reflexões sobre desejo sexual, beleza e feminilidade. SciELO, Saúde e Sociedade , https://www.scielo.br/j/sausoc/a/ZQXKfnnxtSW3FBkTFqM86MB/abstract/?lang=pt. 
Research, Society and Development, v. 11, n. 2, e29811225816, 2022

(CC BY 4.0) | ISSN 2525-3409 | DOI: http://dx.doi.org/10.33448/rsd-v11i2.25816

Vanetti, J. (2017). Untitled. eduCAPES, Diário da Teoria e Prática na Enfermagem, https://educapes.capes.gov.br/bitstream/capes/564218/1/EBOOK\%20Diario\%20da\%20Teoria\%20e\%20Pratica\%20na\%20Enfermagem\%203.pdf.

Vieira, F, et al. (2010). Diagnósticos de enfermagem da NANDA no período pós-parto imediato e tardio. SciELO, Escola Anna Nery 2010, https://www.scielo.br/j/ean/a/btHkypj68Y7w3JPG8JwrzFn/?lang=pt.

Vieiraa, F, et al. (2011). Redalyc.Diagnósticos de enfermagem relacionados à amamentação no puerpério imediato. Redalyc, 2011, https://www.redalyc.org/pdf/3240/324027976003.pdf.

Zimmermmann, J. et al. (2009). Rmmg. Revista Médica de Minas Gerais, http://rmmg.org/sumario/39. 